\title{
Step Detector and Step Length Estimator for an Inertial Pocket Navigation System
}

\author{
Estefania Munoz Diaz and Ana Luz Mendiguchia Gonzalez \\ German Aerospace Center (DLR) \\ Institute of Communications and Navigation \\ Oberpfaffenhofen, 82234 Wessling, Germany \\ Email: Estefania.Munoz@dlr.de
}

\begin{abstract}
The use of smartphones for pedestrian positioning applications has become important in recent years. Algorithms for detecting steps and estimating their length can be found throughout the literature. In this paper we present new algorithms that increase the accuracy of the estimated step length and decrease the number of undetected steps. Unlike state of the art algorithms, that use the acceleration to detect steps and the frequency or the acceleration to estimate the step length, we propose the use of the opening angle of the pedestrian's leg. An experiment with 18 volunteers was conducted in order to prove the relationship between the opening angle of the leg and the step length. Our results show that the estimation of the step length is robust against speed changes and the trajectory's estimated length has less than $1 \%$ error. Our step detector outperforms state of the art algorithms by presenting a higher step detection rate in challenging scenarios. In conclusion, it is possible and convenient to make use of the opening angle of the leg and its relationship with the step length.
\end{abstract}

Index Terms-Pedestrian, dead reckoning, pitch, opening angle of the leg, gait.

\section{INTRODUCTION}

The market for pedestrian positioning applications is rising in recent years. So far, most positioning applications are based on global navigation satellite systems. However, due to the lack of visible satellites, their availability is degraded in certain scenarios such as urban canyons or indoors. The Inertial Measurement Units (IMUs) are a promising self-contained technology to fill this gap.

Commercial and professional users can benefit of this technology, because IMUs are widely accessible. For example, they are embedded in today's smartphones. Therefore, an increasing number of research institutes and companies develop pedestrian navigation systems [1]-[5].

The dead-reckoning algorithm estimates the current user's position for each detected step based on the previous position and the estimation of the step length and the user's heading. In this paper we focus on the estimation of the step length.

To this end, first the step should be detected. There are three mainly used approaches to do it:

- Considering the peaks detection of the pelvis' vertical displacement [6]-[8].

- Detecting foot steps finding peaks in the variance of the vertical acceleration and introducing a threshold to avoid spurious step detections [2].

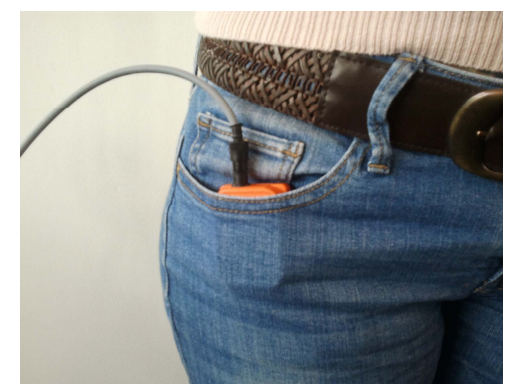

Fig. 1. Our navigation system requires the IMU in the trousers' pocket.

- Evaluating the norm of the complete acceleration for finding peaks by using thresholds as well [9]-[12].

If the complete acceleration is used, the step detection is independent of the sensor's orientation. The peaks correspond to the step occurrences because they are generated by the vertical impact when the foot hits the floor. Since the pattern of impact signal depends on the type of movement and it is greatly affected by the user's walking velocity, the determination of the thresholds for reliable step detection is challenging.

Regarding the step length estimation, the main current techniques can be classified depending on the IMU's location, as specified in [13], [14].

If the IMU is attached to the body near the center of mass:

- Based on a biomechanical model, where the kneeless biped is modeled as an inverted pendulum, and scaling the final estimation with a constant $K$ to calibrate for each user [6].

$$
l_{1}=K \cdot \sqrt{2 L h-h^{2}}
$$

where $l_{1}$ represents the estimated step length, $h$ the vertical displacement of the pelvis and $L$, the leg's length.

- Using an empirical relationship of the vertical acceleration and the step length [7], [9], [15].

$$
l_{2}=K \cdot \sqrt[4]{a_{\max _{v_{i}}}-a_{\min _{v_{i}}}},
$$

where $l_{2}$ represents the estimated step length, $K$ is a calibration value and $a_{\max _{v_{i}}}$ and $a_{\min _{v_{i}}}$ are the maximum and minimum values of the vertical acceleration during step $i$. 
If there are no restrictions on the IMU's placement [4], [10], [11], [16], [17], taking advantage of the relationship between step length $(l)$, height $(h)$, step frequency $\left(f_{\text {step }}\right)$ and some calibration parameters $(a, b, c)$ different for each user, the step length can be estimated as:

$$
l_{3}=h \cdot\left(a \cdot f_{\text {step }}+b\right)+c .
$$

In this paper we present novel algorithms that increase the accuracy of the estimated step length and decrease the number of undetected steps. We propose to make use of the movement of the leg, particularly by exploiting the information of the opening angle of the leg. To this end, we introduce the IMU in the trousers' pocket (Figure 1).

\section{Opening ANGLE OF THE LEG}

In our previous work [5], we have developed an attitude and heading estimator which tracks the orientation of the IMU. The Euler angles are the most intuitive way of describing the orientation of an object, particularly that of a body frame with respect to the corresponding local navigation frame [18]. In our particular case, we do not use quaternions because, with the IMU in the pocket, the dead-lock does not happen. The pitch and roll angles do not reach 90 degrees for a normal way of walking.

At this particular location of the IMU, the yaw and the pitch angles have a remarkable meaning. The yaw or heading angle describes the course of the user, which is well known, and the pitch angle describes the opening angle of the user's leg. We have found this a valuable information and we have used it as the basis of the algorithms described in this paper.

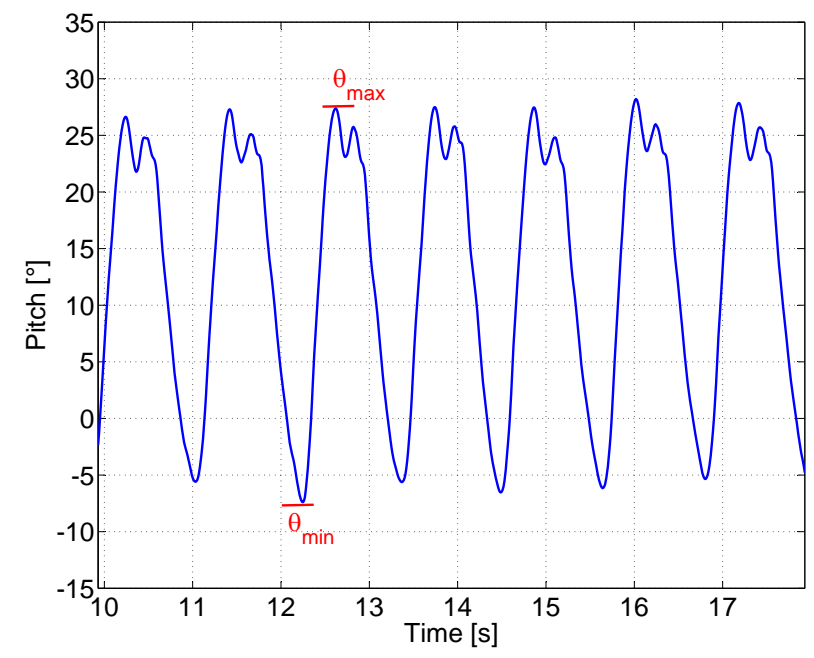

Fig. 2. The blue line represents the opening angle of the leg (pitch angle) during a walk. In this figure, 7 steps are represented.

In Figure 2 the pitch obtained from a pedestrian walking with the IMU in the trouser's pocket is shown. In the figure, a total of 7 steps are represented. This demonstrates the cyclic nature of the steps during the walk, being a pattern easily identifiable. The highest positive peak, $\theta_{\max }$, corresponds to the maximum leg's elongation when walking and the second

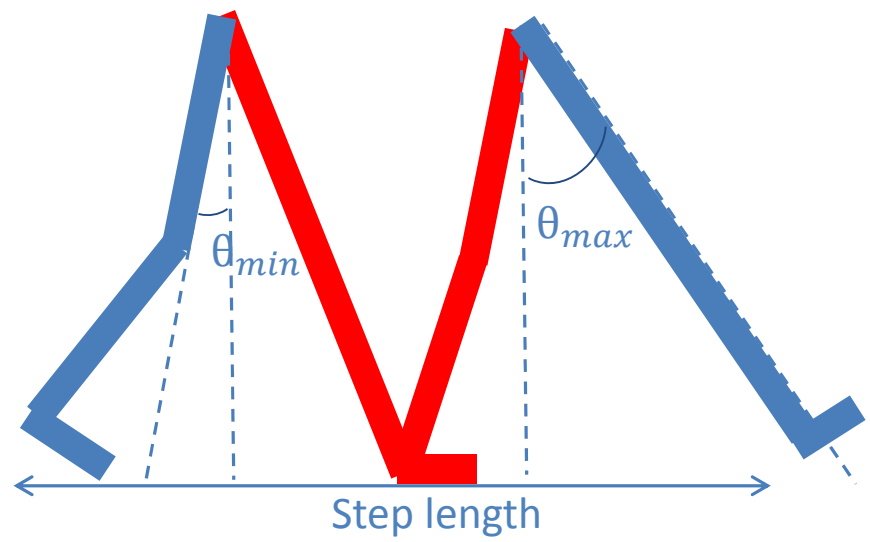

Fig. 3. This figure represents the legs during the occurrence of a step. A step is considered every time the leg which has the IMU, in this case represented in blue, hits the floor. This picture clarifies the angles $\theta_{\max }$ and $\theta_{\min }$.

positive peak occurs as a consequence of the foot hitting the floor. The lowest and unique negative peak is described as $\theta_{\min }$. Figure 3 represents the two legs describing a step. The blue leg carries the IMU in the pocket and a step is considered between hits of the floor of this leg. The vertical dashed line represents a pitch equal to zero. When the pitch angle crosses first to zero in Figure 2 the blue leg overtakes the red leg. When the user is standing the legs are closed, thus the opening angle is zero. By seing Figure 3, it is clear that, because of the knee, $\theta_{\max }$ is much greater than $\theta_{\text {min }}$.

\section{Step Detector}

The pedestrian dead reckoning algorithm estimates the new position of the user every time a new step is detected.

The novel step detector we propose in this work exploits the advantages of tracking the movement of the leg. The opening angle of the leg or pitch is more suitable than the so far used acceleration signal because it describes the movement of the leg in the forward direction, therefore it is consequently related with the occurrence of the steps.

Figure 4 shows the pitch angle represented with the blue line and the green dots represent the detected steps. As it can be seen, the steps are accurately detected with this method.

The used amplitude threshold for the peak detection of the pitch angle is fixed and it does not vary with the user's velocity. This is one of the most important advantages of the proposed approach. Additionally, the signal of the pitch angle is clear enough and does not require any preprocessing or low-pass filtering, in contrast to the case of the step detector based on the norm of the acceleration. The performance of this step detector in challenging scenarios respect to the state of the art approach will be shown in Section V.

When the IMU is handheld with the swinging motion, the behaviour of the pitch angle is similar, not representing the opening angle of the leg, but the swinging angle of the arm. In this case, the approach we propose is also appropriate. 


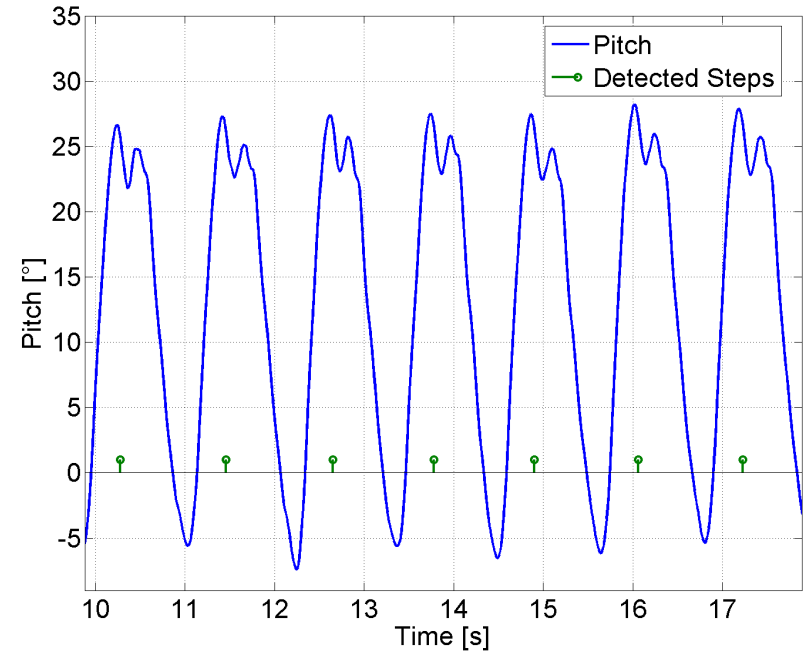

Fig. 4. The blue curve represents the opening angle of the leg (pitch angle) and the detected steps are marked with green dots.

\section{Step Length Estimator}

As mentioned in the introduction, the main techniques used for the estimation of the step length exploit the relationship between step length and step frequency or the step length and the acceleration.

The main motivation to introduce the opening angle of the leg for the step length estimation is that it is highly coupled with the step length. The hypothesis we investigate is that, for the same user, every opening angle of the leg corresponds to a different step length.

Therefore, we have carried out an extensive set of experiments in order to assess this hypothesis. With these experiments we prove that the opening angle of the leg has a relationship with the step length. We test if this relationship can be applied in general for all users and if different users provide similar results. Further, we test if the impact on the step length of other variables such as the user's height, weight, gender and the pocket's height or the user's leg length is relevant.

\section{A. Experimental Set Up}

As previously mentioned, we estimate the pitch with the attitude and heading estimator detailed in [5]. However, for this experiment we have decided to use 16 infrared cameras located on the ceiling of a $18 \mathrm{~m}^{2}$ room. These cameras capture with sub-millimeter accuracy the position and the orientation of the object in the room.

This solution offers several advantages. The first one is that the data for deriving the model will not contain any biases from our commercial IMU or any error due to the raw data process with our attitude and heading estimator. The second advantage is that, due to the sub-millimeter accuracy of the measurement system both, the step length and the pitch angle will be considered for these experiments error free.

We have created a virtual $3 D$ object by sticking three reflectors to our IMU (see Figure 5). Then, we have sewn

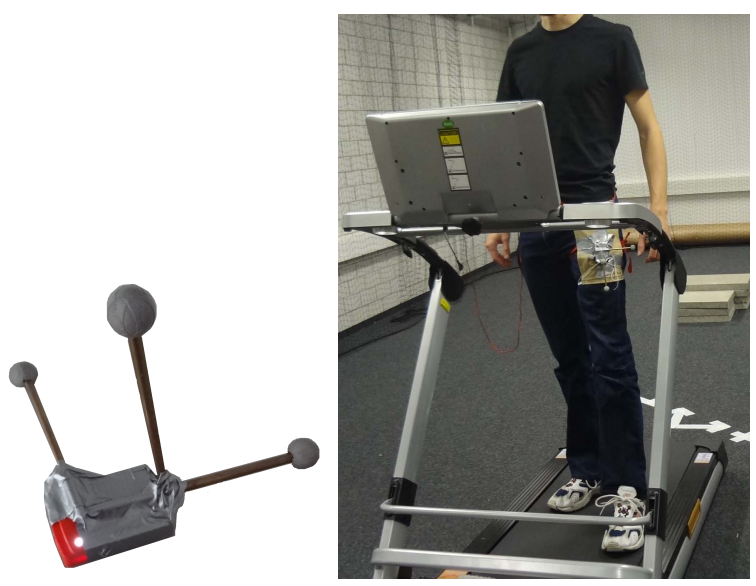

(a) Reflectors on the IMU

(b) External pocket for the IMU

Fig. 5. The infrared cameras on the ceiling capture the movement of the three orthogonal reflectors while the user walks on the running machine. This simulates the movement of the IMU inside the user's pocket.

an external fabric pocket to allocate the IMU at the normal pocket place without introducing it in the real trousers' pocket since this is not possible with the reflectors (see Figure 5). The measurement system tracks the $3 D$ object created with the reflectors at $100 \mathrm{~Hz}$ rate and gives an output of its position and attitude.

Since the room where the measurement system has been installed is too small for our purpose, we have decided to use a running machine. The running machine allows the user to go over long distances within the room. On the other hand, the running machine offers the possibility to select a constant velocity between a set of seven different velocities measured in kilometers per hour. In order to accurately know the user's velocity, reflectors were put on the running machine to compute the ground truth velocity with the infrared cameras' system. This experiment was done for all velocities with a volunteer on the running machine for taking into account the possible modification of the velocity with the weight of a person. Table I shows the different running machine velocities and the postprocessed velocities.

TABLE I

RUNNING MACHINE VELOCITIES

\begin{tabular}{|c|c|c|}
\hline $\begin{array}{c}\text { Running machine } \\
\text { velocity }[\mathrm{km} / \mathrm{h}]\end{array}$ & $\begin{array}{c}\text { Nominal } \\
\text { velocity }[\mathrm{m} / \mathrm{s}]\end{array}$ & $\begin{array}{c}\text { Measured } \\
\text { velocity }[\mathrm{m} / \mathrm{s}]\end{array}$ \\
\hline 1 & 0.2778 & 0.3025 \\
\hline 2 & 0.5556 & 0.607 \\
\hline 3 & 0.8333 & 0.9131 \\
\hline 4 & 1.111 & 1.2132 \\
\hline 5 & 1.3889 & 1.5002 \\
\hline 6 & 1.6667 & 1.8122 \\
\hline 7 & 1.9444 & 2.1244 \\
\hline
\end{tabular}

The experiment counted with 18 volunteers, eight women and ten men with ages between 22 and 57. Firstly, they were asked to provide personal information such as their gender, age and weight. Their height and pocket's height were measured taking into account the height of the shoes' sole. They were 
asked to walk on the running machine and increase the velocity each three minutes until $7 \mathrm{~km} / \mathrm{h}$. For $1 \mathrm{~km} / \mathrm{h}$ and $2 \mathrm{~km} / \mathrm{h}$, volunteers did not feel comfortable on the running machine because of the extremely low velocity. Therefore, we have discarded them for the study. On the other hand, the $7 \mathrm{~km} / \mathrm{h}$ data has been also discarded for the analysis, because some volunteers started running and this is out of the scope of this study.

In order to verify that the running machine does not introduce any anomaly in the way of walking, a subgroup of volunteers was selected to repeat the same experiment but without running machine. For this second experiment, there was a real displacement of the user and the step length could be directly computed without the need of a predefined velocity. For the step detection in both cases, with and without running machine, we have used the detector proposed in Section III.

\section{B. Model Extraction}

More than 15000 steps were recorded during the experiment. This data has been analyzed per volunteer yielding the confirmation of our initial hypothesis: there is a relationship between the opening angle of the leg and the step length. Figure 6 shows the results for one volunteer. Where pitch amplitude is defined as

$$
\Delta \theta=\theta_{\max }-\theta_{\min }
$$

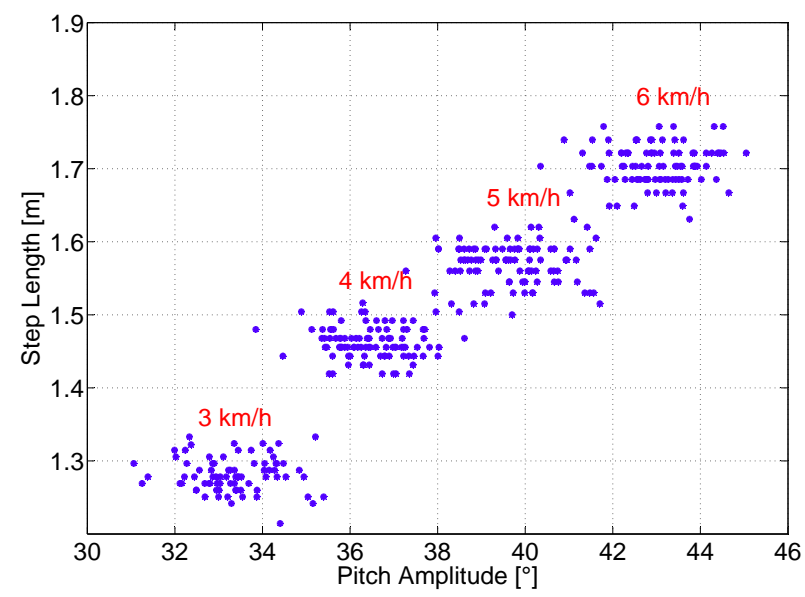

Fig. 6. The points represent the steps from data gathered for one volunteer and each cloud of points represents a different velocity. For every step the pitch amplitude is plotted against the step length.

Figure 6 represents the pitch amplitude in degrees against the step length in meters. Each cloud of points represents a different velocity and each point represents a step. This figure shows the clear trend of the data obtained indicating that, the bigger the opening angle of the leg is, the larger the step length will be. In the figure it can also be seen that the higher the user' velocity is, the larger the step length will be and consequently the greater the opening angle of the leg will be. This can be noticed because the steps recorded for every velocity are sorted increasingly.
Moreover, it has been confirmed that this relationship exists for all volunteers participating in the experiments. In Figure 7 the data for nine different volunteers has been depicted. Each color represents a different volunteer, within the color each cloud of points represents a velocity and each point represents a step.

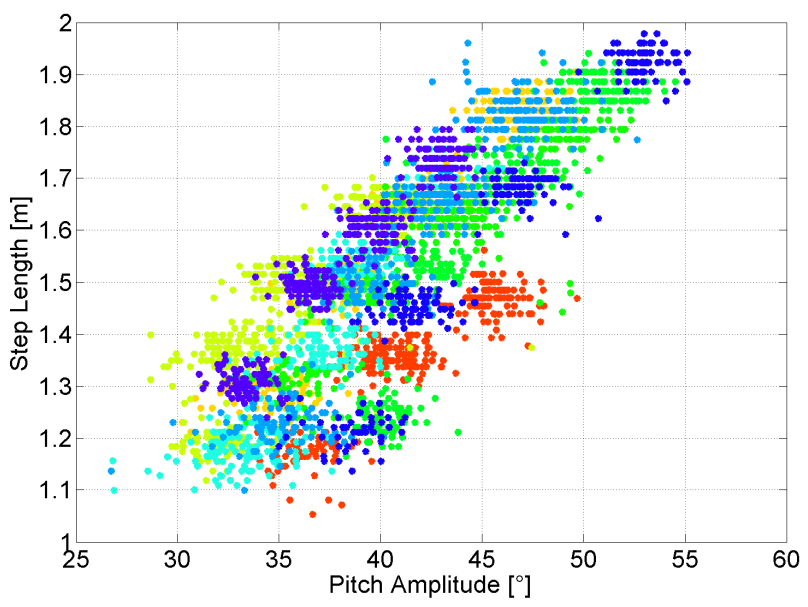

Fig. 7. The points represent the steps from data gathered for nine different volunteers and each color represents a volunteer. For every step the pitch amplitude is plotted against the step length.

Figure 8 shows in dark blue the points of nine volunteers recorded with the running machine (shown in Figure 7) and in light blue the points of five selected volunteers walking without running machine. This demonstrates that the data walking directly on the floor follows the same trend and, consequently, the running machine does not introduce any modification. We can observe more points in the lower part that correspond with lower velocities that we have discarded in the running machine data because the volunteers did not feel comfortable.

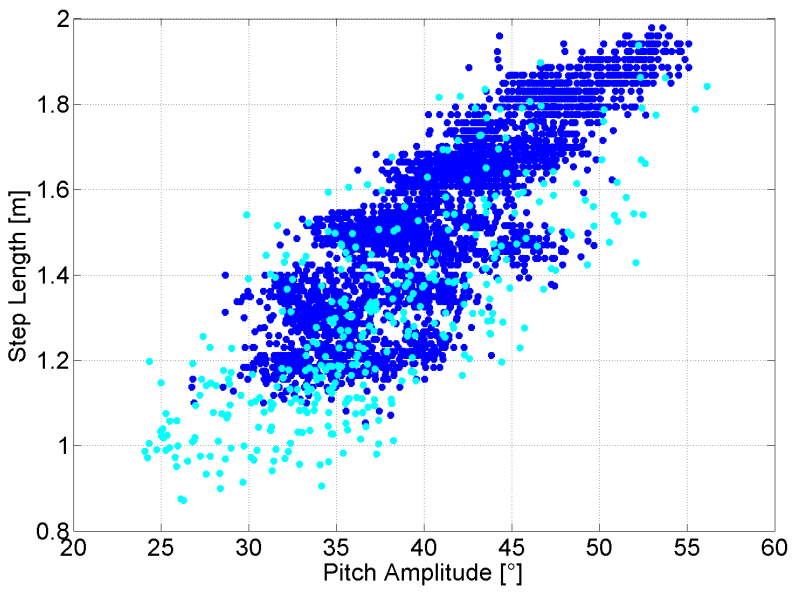

Fig. 8. The dark blue points represent the data recorded with the running machine and the light blue points were recorded walking directly on the floor.

After a first evaluation of the data set, it is rather hard to find a clear relationship with the personal parameters of the 
volunteers and their step length. The regression lines of the volunteers are not sorted by height, pocket's height or weight and they are not grouped by gender either. Even though from the logical point of view the height should have an impact on the step length, the way of walking of each user masks the personal parameters in a way that, makes it not possible to find a conclusion.

Therefore, it has been decided to do a first approximation of the model by computing the regression line for each volunteer. The linear regression is calculated with least squares. The best fit in the least-squares sense minimizes the sum of squared residuals, being a residual the difference between the measured step length and the fitted value provided by the model, the estimated step length.

Therefore, the model for the step length yields:

$$
S L=a \cdot \Delta \theta+b,
$$

where $S L$ is the estimated step length measured in meters taking into account the pitch amplitude, $\Delta \theta$, in degrees. The constants $a$ and $b$ are the personalized parameters fitting each regression line.

\section{EXPERIMENTAL RESUlTS}

\section{A. Step Detector Assessment}

In order to test the novel step detector based on the opening angle of the leg, a volunteer is asked to walk normally (between $3 \mathrm{~km} / \mathrm{h}$ and $6 \mathrm{~km} / \mathrm{h}$ ) with the IMU in the pocket for 3 minutes. The walk started at the second floor of our office building and includes different user's speeds and stairs up to the third floor and down for going back to the initial point. This walk is a challenging scenario for the detectors, since it includes stairs and speed changes, well known weak points for the step detectors. At the same time that the experiment took place, the steps were manually counted.

One of the state of the art approaches for step detection, as explained in the introduction, uses the norm of the acceleration measured by the sensor. Unlike the vertical component, the norm of the acceleration allows to obtain a detection independent of the IMU's orientation. This last procedure has been used in this study for comparing it with the proposed step detector. After implementing it we realized that the norm of the raw acceleration is not appropriate to detect steps accurately with a simple peak detection. Even using amplitude and time thresholds, the number of false detections is prohibitively high. Thus, a preprocess of the signal is required. We have decided to do a 25 samples low-pass filter. The filter smoothes the signal. An amplitude threshold is used in order to avoid false detections. The main problem of using such a threshold for an accurate detection is its dependency with the user's speed.

For the described walk, when the volunteer goes faster (not running) or upstairs and downstairs, steps are missing yielding $87 \%$ of detections. On the other hand, with the novel proposed detector all steps are counted. Similar results have been obtained with 3 more volunteers repeating the same described walk. According to the evaluation of both step detectors, the benefits of our step detector respect to the state of the art one are noticeable. It is easier to implement and provides also better results in challenging scenarios.

\section{B. Step Length Estimator Assessment}

For the evaluation of the step length model, an experiment has been carried out. For this experiment, the volunteer whose data is shown in Figure 6 was asked to walk along a known path of 69 meters, turn 180 degrees and repeat the same path a total of 10 times. The volunteer changed his speed for the different realizations of the straight path between $3 \mathrm{~km} / \mathrm{h}$ and $6 \mathrm{~km} / \mathrm{h}$. Every time the volunteer turns 180 degrees his speed decreases drastically. The results are summarized in Table II.

TABLE II

STEP LENGTH ASSESSMENT

\begin{tabular}{|c|c|c|}
\hline $\begin{array}{c}\text { Estimated Distance }[m] \\
\text { Mean }\end{array}$ & $\begin{array}{c}\text { Estimated Distance }[m] \\
\text { Std. Dev. }\end{array}$ & $\begin{array}{c}\text { Error [\%] } \\
\text { Mean }\end{array}$ \\
\hline 69.1 & 1.09 & 0.15 \\
\hline
\end{tabular}

For obtaining these results the model described in Equation 4 has been used. The parameters $a$ and $b$ are the volunteer's personalized parameters extracted from his data showed in Figure 6. No pre-calibration before this experiment has been carried out. These results show an excellent performance of the model of the step length based on the opening angle of the leg with an estimated distance of mean 69.1 meters, yielding a mean error of $0.15 \%$. The 10 realizations of the straight line of 69 meters at different speeds have a standard deviation of 1 meter, therefore the derived model offers good results even when the user does not walk at a constant speed.

It is worthy to mention that this experiment does not reflect the error in the individual step length estimation, but the error for the complete trajectory of 69 meters. It is clear that little over- and underestimations of the individual step length estimations are compensated. However, we are interested in showing this result because for navigating a user, an accurate trajectory, i.e. the sum of all steps, makes the difference.

\section{CONClusions AND OUtLOOK}

The objective of this paper is to present new algorithms to estimate the step length and to detect steps for pedestrian deadreckoning navigation systems. Although in the literature such algorithms exist, this paper aims at decreasing, in the case of the step detector, the number of false detections and undetected steps. In the case of the step length estimator, this paper aims at providing a significant improvement in the accuracy of the estimated length.

We present a step detector and step length estimator based on the opening angle of the leg. We demonstrate that the movement of the user's leg is highly coupled with the step length and the occurrence of steps. We carry out an experiment for deriving a model for the step length with a sub-millimeter accuracy measurement system.

We compare the performance of our step detector against one of the state of the art step detectors and we demonstrate 
the benefits of using the novel detector proposed in this work. Regarding the step length estimator, we assess it by doing a stress test walking at different speeds including also fast changes of speed.

As an outlook, we consider a natural continuation of this work to do a comparative study of the novel step length estimator proposed in this work with the main estimators proposed in the literature. The possibility of complementing the state of the art estimator using the frequency with the estimator proposed in this work should be studied. We also think it is possible to deduce a biomechanical model for the estimation of the step length based on the opening angle of the leg.

\section{ACKNOWLEDGEMENTS}

The authors would like to thank Fabian de Ponte Müller for his help and the volunteers of the experiments for their patience and goodwill.

\section{REFERENCES}

[1] J. W. Kim, H. J. Jang, D.-H. Hwang, and C. Park, "A step, stride and heading determination for the pedestrian navigation system," Journal of Global Positioning Systems, vol. 3, no. 1-2, pp. 273-279, 2004.

[2] U. Steinhoff and B. Schiele, "Dead reckoning from the pocket-an experimental study," in Pervasive Computing and Communications (PerCom), 2010 IEEE International Conference on. IEEE, 2010, pp. 162-170.

[3] A. Jimenez, F. Seco, C. Prieto, and J. Guevara, "A comparison of pedestrian dead-reckoning algorithms using a low-cost mems imu," in Intelligent Signal Processing, 2009. WISP 2009. IEEE International Symposium on. IEEE, 2009, pp. 37-42.

[4] V. Renaudin, M. Susi, and G. Lachapelle, "Step length estimation using handheld inertial sensors," Sensors, vol. 12, no. 7, pp. 8507-8525, 2012.

[5] E. Munoz Diaz, A. L. Mendiguchia Gonzalez, and F. de Ponte Müller, "Standalone Inertial Pocket Navigation System," IEEE/ION Position Location and Navigation Symposium, Monterey, USA, pp. 241-251, May 2014.

[6] W.-Y. Shih, L.-Y. Chen, and K.-C. Lan, "Estimating walking distance with a smart phone," in Parallel Architectures, Algorithms and Programming (PAAP), 2012 Fifth International Symposium on. IEEE, 2012, pp. 166-171.

[7] P. Goyal, V. J. Ribeiro, H. Saran, and A. Kumar, "Strap-down pedestrian dead-reckoning system," in Indoor Positioning and Indoor Navigation (IPIN), 2011 International Conference on. IEEE, 2011, pp. 1-7.

[8] C.-C. Lo, C.-P. Chiu, Y.-C. Tseng, S.-A. Chang, and L.-C. Kuo, "A walking velocity update technique for pedestrian dead-reckoning applications," in Personal Indoor and Mobile Radio Communications (PIMRC), 2011 IEEE 22nd International Symposium on. IEEE, 2011, pp. 1249-1253.

[9] Y. Jin, H.-S. Toh, W.-S. Soh, and W.-C. Wong, "A robust dead-reckoning pedestrian tracking system with low cost sensors," in Pervasive Computing and Communications (PerCom), 2011 IEEE International Conference on. IEEE, 2011, pp. 222-230.

[10] S. Shin, M. Lee, C. Park, and H. S. Hong, "Pedestrian dead reckoning system with phone location awareness algorithm," in Position Location and Navigation Symposium (PLANS), 2010 IEEE/ION. IEEE, 2010, pp. 97-101.

[11] D. Gusenbauer, C. Isert, and J. Krosche, "Self-contained indoor positioning on off-the-shelf mobile devices," in Indoor Positioning and Indoor Navigation (IPIN), 2010 International Conference on. IEEE, 2010, pp. 1-9.

[12] M. Alzantot and M. Youssef, "Uptime: Ubiquitous pedestrian tracking using mobile phones," in Wireless Communications and Networking Conference (WCNC), 2012 IEEE. IEEE, 2012, pp. 3204-3209.

[13] D. Alvarez, R. C. González, A. López, and J. C. Alvarez, "Comparison of step length estimators from weareable accelerometer devices," in Engineering in Medicine and Biology Society, 2006. EMBS'06. 28th Annual International Conference of the IEEE. IEEE, 2006, pp. 59645967.
[14] J. Jahn, U. Batzer, J. Seitz, L. Patino-Studencka, and J. Gutiérrez Boronat, "Comparison and evaluation of acceleration based step length estimators for handheld devices," in Indoor Positioning and Indoor Navigation (IPIN), 2010 International Conference on. IEEE, 2010, pp. 1-6.

[15] Z. Yu, "Dead reckoning algorithms for indoor localization," Master's thesis, National University, Singapore, 2012.

[16] V. Renaudin, V. Demeule, and M. Ortiz, "Adaptative pedestrian displacement estimation with a smartphone," internacional Conference on Indoor Positioning and Indoor Navigation, vol. 12, pp. 916-924, 2013.

[17] S. Shin, C. Park, J. Kim, H. Hong, and J. Lee, "Adaptive step length estimation algorithm using low-cost mems inertial sensors," in Sensors Applications Symposium, 2007. SAS'07. IEEE. IEEE, 2007, pp. 1-5.

[18] P. Groves, Principles of GNSS, Inertial, and Multisensor Integrated Navigation Systems, Second Edition:, ser. GNSS/GPS. Artech House, 2013. 